Article

\title{
Multifunctional Platform with CMOS-Compatible Tungsten Microhotplate for Pirani, Temperature, and Gas Sensor
}

\author{
Jiaqi Wang * and Jun Yu \\ Received: 26 July 2015 ; Accepted: 20 October 2015 ; Published: 28 October 2015 \\ Academic Editor: Ching-Liang Dai \\ Key Laboratory of Liaoning for Integrated Circuits Technology, School of Electronic Science and Technology, \\ Dalian University of Technology, Dalian 116024, China; junyu@dlut.edu.cn \\ * Correspondence: wjq@dlut.edu.cn; Tel.: +86-411-8470-6184; Fax: +86-411-8470-6706
}

\begin{abstract}
A multifunctional platform based on the microhotplate was developed for applications including a Pirani vacuum gauge, temperature, and gas sensor. It consisted of a tungsten microhotplate and an on-chip operational amplifier. The platform was fabricated in a standard complementary metal oxide semiconductor (CMOS) process. A tungsten plug in standard CMOS process was specially designed as the serpentine resistor for the microhotplate, acting as both heater and thermister. With the sacrificial layer technology, the microhotplate was suspended over the silicon substrate with a $340 \mathrm{~nm}$ gap. The on-chip operational amplifier provided a bias current for the microhotplate. This platform has been used to develop different kinds of sensors. The first one was a Pirani vacuum gauge ranging from $10^{-1}$ to $10^{5} \mathrm{~Pa}$. The second one was a temperature sensor ranging from -20 to $70^{\circ} \mathrm{C}$. The third one was a thermal-conductivity gas sensor, which could distinguish gases with different thermal conductivities in constant gas pressure and environment temperature. In the fourth application, with extra fabrication processes including the deposition of gas-sensitive film, the platform was used as a metal-oxide gas sensor for the detection of gas concentration.
\end{abstract}

Keywords: multifunctional platform; microhotplate; Pirani sensor; temperature sensor; gas sensor

\section{Introduction}

Recently, complementary metal oxide semiconductor (CMOS) compatible sensors have been widely used in the detection of pressure, temperature, acceleration, and chemical leakage [1-4]. With better control and optimization of CMOS technology, the manufacturing yield of the sensors can be dramatically increased [5]. The noise level of the sensor would be decreased by integrating the sensor and the signal processing circuit in one chip [6]. With the fast development of various kinds of sensors, the idea of multifunctional platforms for the different sensors is generated [7]. With these multifunctional platforms, different kinds of sensors can be implemented, which would save a lot of development time and cost.

The microhotplate is a good multifunctional platform. It includes the thin membrane for pressure measurement, the heater and thermister for thermal-related applications, and the free-standing structure with excellent thermal isolation for high temperature platforms. The microhotplate fabricated by microelectromechanical systems (MEMS) technology has been widely used for gas pressure sensors, gas sensors, chemical sensors, flow sensors, and accelerometers [8-13]. It could also be implemented in the standard CMOS process for high yield and low cost. The most important considerations for the CMOS-compatible microhotplate fabrication include the CMOS-compatible materials and the post-CMOS process. The materials of microhotplate mainly 
include the heating material and the thermal insulation material. Many different heating materials are available for microhotplate, such as polysilicon [14], aluminum (Al) [15], platinum (Pt) [16], and tungsten (W) [17]. Platinum has been considered the best material for the microhotplate, but it is not the material in the standard CMOS process. Aluminum and polysilicon cannot endure high currents and high temperatures. Tungsten is suggested to be the perfect heating and temperature measurement material for microhotplate due to its properties of high melting point, large temperature coefficient, low cost, and CMOS compatibility. The $\mathrm{SiO}_{2}$ and $\mathrm{Si}_{3} \mathrm{~N}_{4}$ as the dielectric layer in the CMOS process are commonly used as the thermal insulation layer for the microhotplate. The post-CMOS processes for the microhotplate mainly include the etching process to suspend the microhotplate from the substrate. Alkaline solutions are used to etch silicon or polysilicon to suspend the microhotplate. The fact that most of the alkaline solutions attack the aluminum bonding pads should be considered. One way is to protect the aluminum pads with some metal, such as $\mathrm{Pt}$, against alkaline corrosion [18]. The improved tetramethylammonium hydroxide (TMAH) has also been used as an effective method to remove the silicon without damaging the aluminum pads $[19,20]$.

In this paper, tungsten was employed as the heating and temperature measurement material for the microhotplate. An improved TMAH solution was used to remove the polysilicon sacrificial layer to suspend the microhotplate. The multifunctional platform for sensors consisted of the tungsten microhotplate and the on-chip operational amplifier. A bias current for the microhotplate was supplied by the operational amplifier. The platform for sensors was implemented in $0.5 \mu \mathrm{m}$ standard CMOS process. It has been employed to develop a Pirani gas pressure, temperature, and thermal-conductivity gas sensor based on the fact that the gaseous thermal conduction is mainly affected by gas pressure, gas temperature, and the type of the gas. Besides those applications, with a little extra fabrication process, including deposition of the gas-sensing film on the microhotplate, this platform could be used as the heating component for a metal-oxide gas sensor.

\section{Experimental Section}

\subsection{Fabrication Process of Tungsten Microhotplate}

Tungsten has been traditionally used as a plug material to form via pathways between various metal layers due to its ability to uniformly fill the high aspect ratio vias when deposited by chemical vapor deposition (CVD) methods. In our design, the tungsten microhotplate was fabricated in $0.5 \mu \mathrm{m}$ standard CMOS process, which featured two polysilicon layers (Poly1 and Poly2) and two aluminum interconnection layers (Metal1, Metal2). The metal plug between Metal1 and Metal2 was tungsten. Dielectric layers surrounding the metal layers were phosphosilicate glass (PSG). The $\mathrm{Si}_{3} \mathrm{~N}_{4}$ was more stable than PSG so it was used as the passivation layer on the surface to protect the chip. In the design of the tungsten microhotplate, tungsten was in the form of serpentine resistor instead of via plug. The anchors of the tungsten resistor were connected to Metal2, leaving Metal1 unconnected. A $0.34 \mu \mathrm{m}$ thick Poly 2 was used as a sacrificial layer below the tungsten microhotplate. The etch windows of the tungsten microhotplate were opened during the bonding-pad patterning process in a standard CMOS process, as shown in Figure 1a. Figure $1 \mathrm{~b}$ shows that the etch windows for the microhotplate and the bonding pads were etched simultaneously during the bonding pad dry-etching in the CMOS process until Poly2 was exposed. Lastly, in our lab, Poly2 was removed to suspend the tungsten microhotplate by an improved TMAH etchant which avoided etching the exposed aluminum bonding pads. Before starting the etching process, the aluminum layer was sputtered at the backside of the die to keep etching substrate from the backside. It took about $8 \mathrm{~h}$ to remove the sacrificial layer with the improved TMAH etchant in $85^{\circ} \mathrm{C}$, as shown in Figure 1c [21].

\subsection{Multifunctional Platform with Microhotplate and Operational Amplifier}

The constant current circuit was adopted to bias the microhotplate [22], as shown in Figure 2. $R_{1}$ was the tungsten resistor of the microhotplate and $R_{2}$ was the reference resistor in the substrate. 
In Figure 2, $V_{\text {ref }}$ was an external reference voltage and Equation (1) defined the output voltage $V_{\text {out }}$ :

$$
V_{\text {out }}=V_{\text {ref }}\left(1+\frac{R_{1}}{R_{2}}\right)
$$
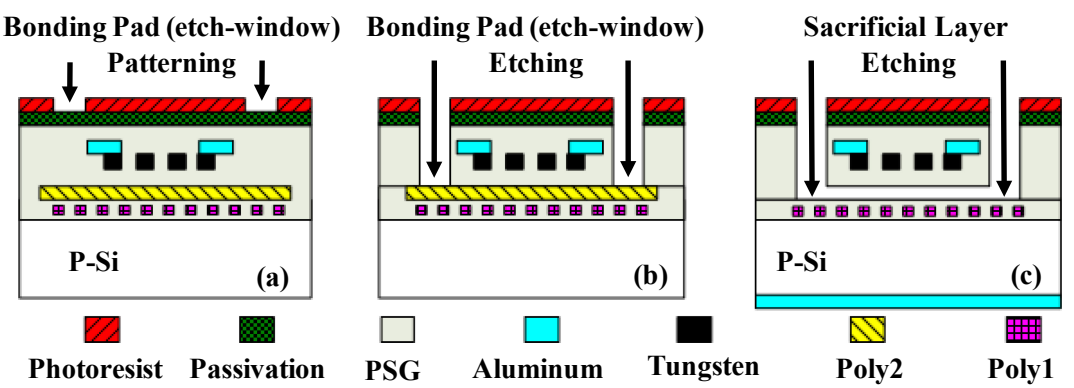

Figure 1. Fabrication of the freestanding structure of the tungsten microhotplate. (a) Opening of the etch windows for the tungsten microhotplate; (b) Dry-etching process to expose the sacrificial layer for the tungsten microhotplate; (c) Suspending of the tungsten microhotplate by the removal of the sacrificial layer.

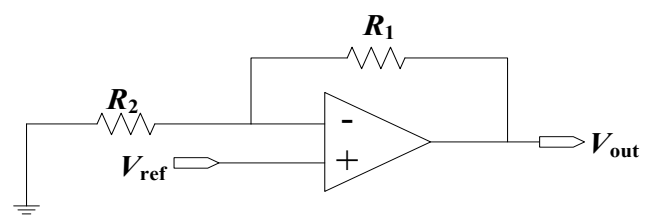

Figure 2. Constant current circuit for the microhotplate.

When the $V_{\text {ref }}$ was set to constant, the voltage in the minus input of the amplifier was also constant. $R_{2}$ was in the substrate whose temperature was considered equal to the ambient temperature. During application of the sensor, the ambient temperature would be controlled to be as stable as possible, so $R_{2}$ was nearly constant and the current through $R_{2}$ was also considered constant. The current through $R_{1}$ was equal to the current of $R_{2}$ since the input current of the ideal amplifier was zero. That temperature compensation technique was required in order to avoid the influence of the ambient temperature fluctuation. For the constant current circuit, $R_{2}$ was also used as the temperature compensation resistor. Considering the influence of ambient temperature fluctuation, the output voltage $V_{\text {out }}$ was defined by Equation (2):

$$
V_{\text {out }}=V_{\text {ref }}\left[1+\frac{R_{1}\left(1+\alpha_{1} \Delta T\right)}{R_{2}\left(1+\alpha_{2} \Delta T\right)}\right]
$$

where $\alpha_{1}$ and $\alpha_{2}$ are the temperature coefficient for resistors in the microhotplate $\left(R_{1}\right)$ and in the substrate $\left(R_{2}\right)$, respectively; $\Delta T$ was the ambient temperature fluctuation. If $R_{1}$ and $R_{2}$ adopted the same material, $\alpha_{1}$ was equal to $\alpha_{2}$. Thus, $R_{2}$ was also a tungsten resistor, but in the substrate, to compensate the influence of the temperature variation of the substrate. In this situation, Equation (2) would change to Equation (1), and the output voltage was not influenced by the ambient temperature fluctuation.

Figure 3 shows the optical and scanning electron microscope (SEM) pictures of the multifunctional sensor platform. The tungsten microhotplate with a square area of $60 \mu \mathrm{m} \times 60 \mu \mathrm{m}$ was suspended by four beams which had a length of $30 \mu \mathrm{m}$ and a width of $15 \mu \mathrm{m}$. The width of the tungsten resistor was $0.8 \mu \mathrm{m}$. The thickness of the microhotplate was about $4 \mu \mathrm{m}$. The gap between the tungsten microhotplate and the substrate was $0.34 \mu \mathrm{m}$ according to the thickness of Poly2 as the 
sacrificial layer. The end of the sacrificial layer (Poly2) removal process could be identified according to the color difference of the Poly1 concentric square after Poly2 removal, as compared in Figure 3a,b. Figure $3 c$ shows the SEM picture of the microhotplate. Figure 3d shows the SEM picture of the microhotplate platform, consisting of the tungsten microhotplate array, the reference resistors, and the constant current circuit with the on-chip operational amplifier.

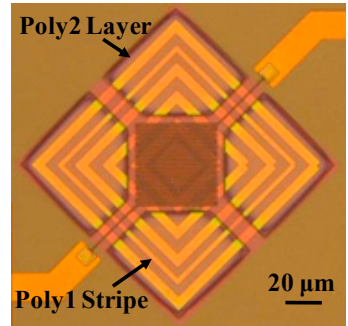

(a)

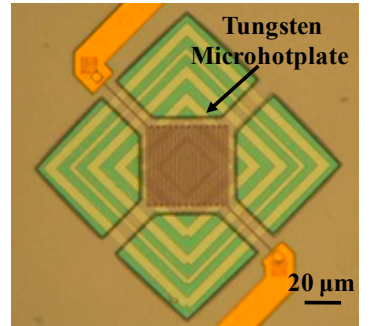

(b)

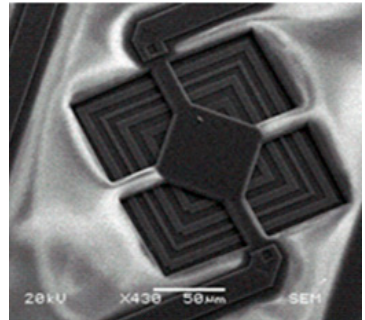

(c)

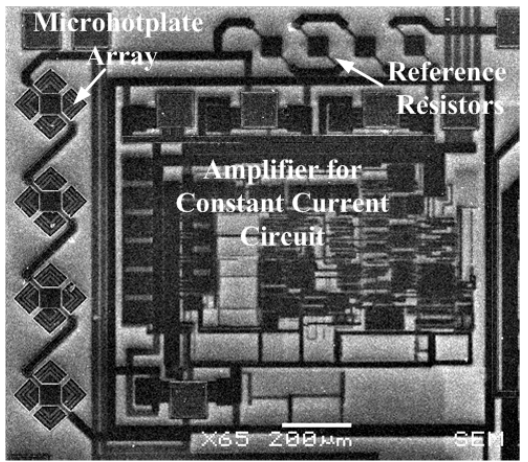

(d)

Figure 3. Pictures of the multifunctional sensor platform. (a) Optical microscopy picture of the tungsten microhotplate before etching; (b) Optical microscopy picture of the tungsten microhotplate after etching; (c) scanning electron microscope (SEM) picture of the tungsten microhotplate; (d) SEM picture of the platform for sensors.

\section{Results and Discussions}

Based on the microhotplate platform, different kinds of sensors were developed. Without any extra fabrication processes, this platform was developed for a Pirani gas pressure sensor, a gas temperature sensor, and a thermal-conductivity gas sensor. With a few fabrication processes, including the deposition of aluminum electrodes and sensitive chemical film, the platform was used as the metal-oxide gas sensor. Thus, experiments were carried out in four aspects. First, for the same type of gas, the gas pressure was measured when the gas temperature was constant. Second, for the same type of gas, the gas temperature was measured when the gas pressure was constant. Third, the responses to the different types of gases with different thermal conductivities were introduced at the constant temperature and gas pressure. Fourth, after deposition of sensitive material on the microhotplate, the concentration of the alcohol gas was detected with the platform. All the results showed that the microhotplate was an effective platform to develop a gas pressure sensor, a gas temperature sensor, a thermal-conductivity gas sensor, and a metal-oxide gas sensor.

\subsection{Measurement of Pirani Gas Pressure Sensor}

The working principle of the Pirani gas pressure sensor is based on the fact that the heat loss of the hotplate to its substrate through gas conduction is a function of the gas thermal conductivity. It is widely used in rough vacuum measurement. In the experiment, the platform configured as the Pirani sensor was put into the vacuum chamber. The temperature in the chamber was $30.3^{\circ} \mathrm{C}$, as measured by a PT100 thermal resistor (Hayashi Denko, Tokyo, Japan). The bias current through 
the microhotplate was $3.85 \mathrm{~mA}$. The measurement procedure was divided into two steps. First, the gas pressure was decreased from atmosphere to $10^{-1} \mathrm{~Pa}$. Second, when the gas pressure of the vacuum system was below $1 \mathrm{~Pa}$, the pump was isolated, then the air slowly bled into the chamber to the desired pressure, and the real-time output voltage of the circuit was recorded with a personal computer using the A/D card. Figure 4 shows the typical response curve of the sensor to the gas pressure ranging from $10^{-1}$ to $10^{5} \mathrm{~Pa}$, which made the sensor platform suitable for the Pirani vacuum sensor.

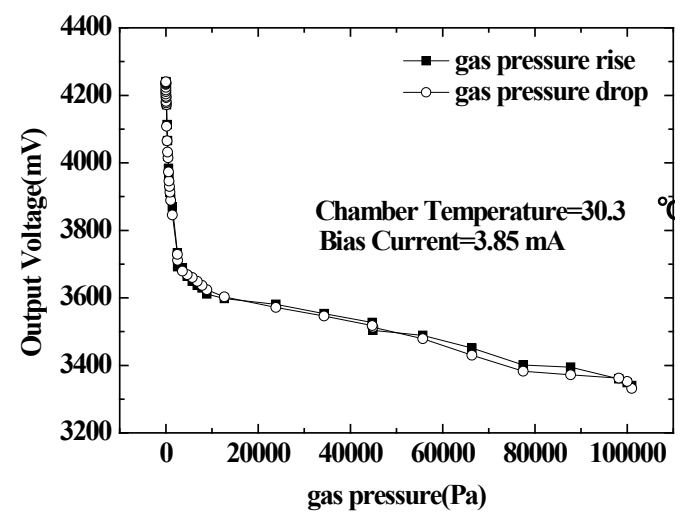

Figure 4. The response of the sensor platform to the gas pressure from $10^{-1}$ to $10^{5} \mathrm{~Pa}$.

According to the measurement results, the whole rough vacuum range could be divided into several ranges. The Pirani sensor biased by constant current had different sensitivities to each vacuum range, as shown in Table 1 . In the high vacuum from 0.1 to $4 \mathrm{~Pa}$, the sensitivity of the sensor was $1.8 \mathrm{mV} / \mathrm{Pa}$, while in the low vacuum from $10^{4}$ to $10^{5} \mathrm{~Pa}$, the sensitivity of sensor decreased to $0.0025 \mathrm{mV} / \mathrm{Pa}$. Thus, the Pirani sensor biased by constant current circuit had high sensitivity in high vacuum.

Table 1. The sensitivity of the Pirani sensor in different vacuum ranges.

\begin{tabular}{cc}
\hline Vacuum Range & Average Sensitivity \\
\hline $0.1-4 \mathrm{~Pa}$ & $1.8 \mathrm{mV} / \mathrm{Pa}$ \\
$4-100 \mathrm{~Pa}$ & $0.7 \mathrm{mV} / \mathrm{Pa}$ \\
$100-1000 \mathrm{~Pa}$ & $0.28 \mathrm{mV} / \mathrm{Pa}$ \\
$1000-3500 \mathrm{~Pa}$ & $0.081 \mathrm{mV} / \mathrm{Pa}$ \\
$3500-10,000 \mathrm{~Pa}$ & $0.013 \mathrm{mV} / \mathrm{Pa}$ \\
$10,000-100,000 \mathrm{~Pa}$ & $0.0025 \mathrm{mV} / \mathrm{Pa}$ \\
\hline
\end{tabular}

\subsection{Measurement of Temperature Sensor}

Thermal conduction of gas is also influenced by the gas temperature, which is the principle of the temperature sensor discussed in detail in our previous research [23]. The gas in the gap between the microhotplate and the substrate was employed as the sensory component for the temperature sensor. In the test, the platform configured as the temperature sensor was placed in the temperature-controlled oven filled with air at atmospheric pressure and the temperature was adjusted each Celsius degree from -20 to $70{ }^{\circ} \mathrm{C}$. The output voltage for the corresponding temperature was recorded by a multimeter. The measurement results were shown in Figure 5. The sensor showed a good linearity over the temperature range, and the temperature coefficient was $0.303 \%$ when the bias current through the microhotplate was $3.85 \mathrm{~mA}$.

The samples without the etching process and suspended gap have also been tested. For these samples, since there was no suspended gap, the tungsten resistor in the microhotplate was also in the silicon substrate. Therefore, as shown in Figure 2, both $R_{1}$ (the resistors in microhotplate) and 
$R_{2}$ (the resistors in substrate) had the same response to the temperature changes. According to Equation (1), the output voltage had little response to the temperature change, measured about $1 \mathrm{mV}$ fluctuation from -20 to $70{ }^{\circ} \mathrm{C}$, probably due to the measurement error, noise, and temperature drift of the operational amplifier, as shown in Figure 5. The obvious difference for the response of the microhotplate with and without the suspended gap was the result of the temperature-related thermal conduction of the gas [23]. As the sensory component was the gas, the inflatable sealed package filled with $\mathrm{N}_{2}$ or inert gas would be adopted as the ideal package for the temperature sensor.

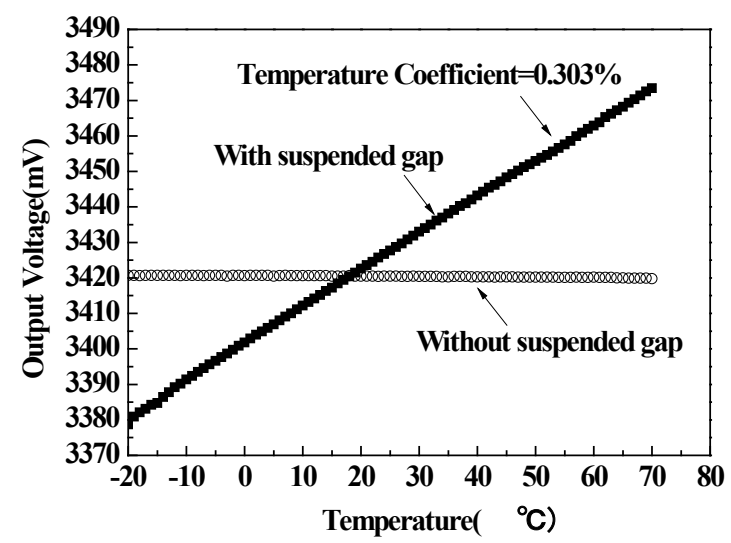

Figure 5. The response of the sensor platform from -20 to $70{ }^{\circ} \mathrm{C}$.

\subsection{Measurement of Thermal-Conductivity Gas Sensor}

For the gases with different thermal conductivities, this platform also had the response due to the different thermal conduction of each gas. The experiment was carried out at atmospheric pressure, in $25^{\circ} \mathrm{C}$. The various gases, including $\mathrm{CH}_{3} \mathrm{CH}_{2} \mathrm{OH}$ (ethanol), $\mathrm{CH}_{3} \mathrm{COOH}$ (acetic acid), $\mathrm{CH}_{3} \mathrm{COCH}_{3}$ (acetone), $\mathrm{NH}_{3}$ (ammonia), and $\mathrm{HCHO}$ (formaldehyde) mixed with air, respectively, were bled slowly to the platform in the chamber with a syringe. Figure 6 presents the responses of the platform to the different gases. The responses to the organic gases were similar due to their similar thermal conductivity. $\mathrm{NH}_{3}$ had larger thermal conductivity compared to the organic gases, so it had an obvious response. When the gas concentration decreased over time, the output voltages of the sensor gradually got closer to the conditions in the air's atmosphere. This experiment was not so precise; nevertheless, the platform could be used for the thermal-conductivity gas sensor, especially for $\mathrm{H}_{2}$ or He detection due to their high thermal conductivity $[24,25]$.

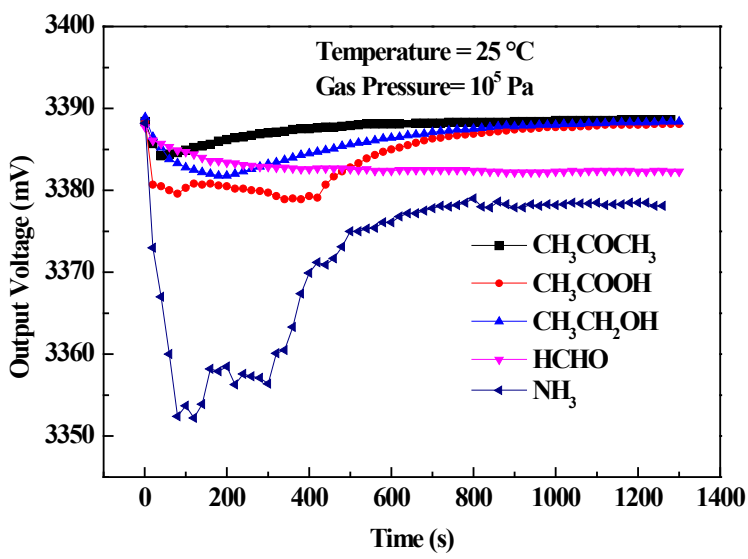

Figure 6. The response of the platform to the gases with different thermal conductivities, including ethanol, acetic acid, acetone, ammonia, and formaldehyde mixed with air at atmosphere pressure, in $25^{\circ} \mathrm{C}$, respectively. 


\subsection{Measurement of Metal-Oxide Gas Sensor}

The metal-oxide gas sensor requires working in high temperature for better performance. This platform could be used as the heating plate for metal-oxide gas sensors with extra chemically sensitive material on the surface of the microhotplate $[6,9,26]$. In our experiment, two aluminum electrodes were fabricated on the surface of the microhotplate during the CMOS process. Thin $\mathrm{SnO}_{2}$ film was sputtered on the microhotplate in our lab as the gas-sensitive material, as shown in Figure 7a. The resistance of the $\mathrm{SnO}_{2}$ film was measured as $0.3 \mathrm{M} \Omega$. During the gas detection measurement, the sensor was placed in a $50 \mathrm{~L}$ gas chamber, and ethanol was injected into the chamber. The resistance of the sensor was recorded by the multimeter. The sensing film was heated up to $300{ }^{\circ} \mathrm{C}$ with the microhotplate platform, and resistance changes of the $\mathrm{SnO}_{2}$ film to ethanol were measured after one week of preheating. Figure $7 \mathrm{~b}$ shows the typical sensing response curve to ethanol. The gas sensor had a good response to ethanol, and the resistance of the sensor increased with the ethanol concentration increasing from 30 to $2000 \mathrm{ppm}$.

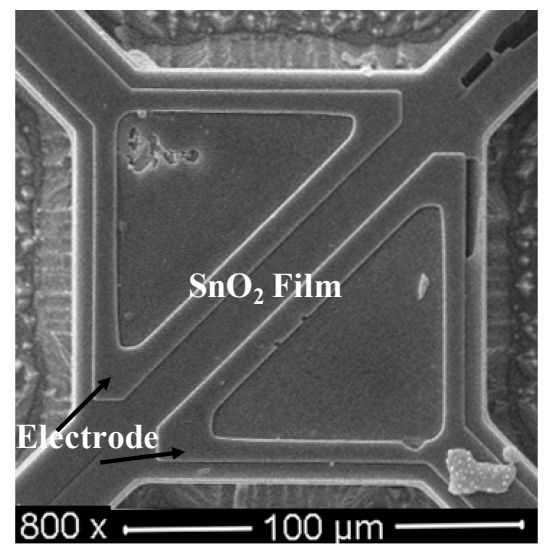

(a)

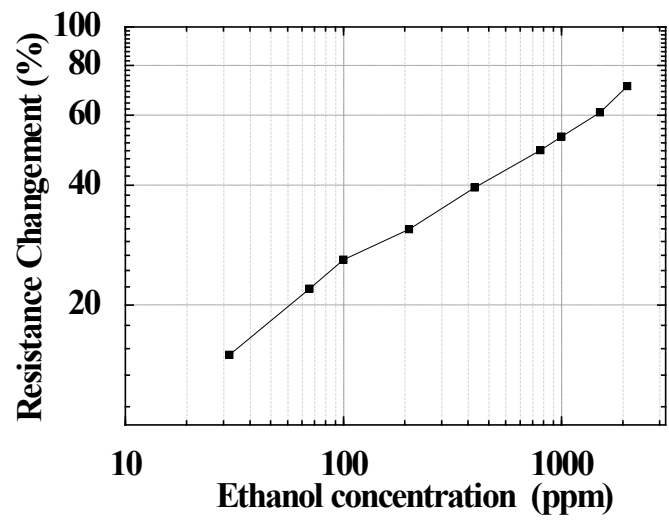

(b)

Figure 7. Microhotplate platform configured as a metal-oxide gas sensor with $\mathrm{SnO}_{2}$-sensitive film and the response to ethanol. (a) Scanning electron microscope (SEM) picture of the tungsten microhotplate for metal-oxide gas sensor; (b) Response of metal-oxide gas sensor to ethanol with different concentrations at $300{ }^{\circ} \mathrm{C}$.

\section{Conclusions}

Many CMOS-compatible sensors have been developed in recent years. For some similar kinds of sensors, it would be effective to develop a uniform platform for them. Based on the same platform, different sensors could be developed with a shortened research cycle, low cost, and similar standard.

In this paper, the multifunctional platform for sensors based on the microhotplate was developed. The tungsten microhotplate could afford high temperature and it was fully CMOS-compatible. Without extra fabrication processes, this platform has been configured as the Pirani gas pressure, temperature, and thermal-conductivity gas sensor, respectively. Besides those applications, with a few extra fabrication processes, including the deposition of the sensory film on the microhotplate, this platform can be employed as the heating component for some metal-oxide gas sensors.

Acknowledgments: This work was supported by the National Natural Science Foundation of China (Nos. 61201035 and 61274076).

Author Contributions: Jiaqi Wang developed the sensor platform and tested the Pirani gas pressure, and temperature sensor. Jun Yu designed the gas sensor test system and tested the gas sensor.

Conflicts of Interest: The authors declare no conflict of interest. 


\section{References}

1. Dai, C.L.; Lu, P.W.; Chang, C.L.; Liu, C.Y. Capacitive micro pressure sensor integrated with a ring oscillator circuit on chip. Sensors 2009, 9, 10158-10170. [CrossRef] [PubMed]

2. Chen, S.J.; Shen, C.H. A novel two-axis CMOS accelerometer based on thermal convection. IEEE Trans. Instrum. Meas. 2008, 57, 1572-1577. [CrossRef]

3. Souri, K.; Chae, Y.; Makinwa, K.A.A. A CMOS temperature sensor with a voltage-calibrated inaccuracy of $\pm 0.15{ }^{\circ} \mathrm{C}(3 \sigma)$ from $-55^{\circ} \mathrm{C}$ to $125^{\circ} \mathrm{C}$. IEEE J. Solid State Circuits 2013, 48, 292-301. [CrossRef]

4. Hagleitner, C.; Hierlemann, A.; Lange, D.; Kummer, A.; Kerness, N.; Brand, O.; Baltes, H. Smart single-chip gas sensor microsystem. Nature 2001, 414, 293-296. [CrossRef] [PubMed]

5. Baltes, H.; Brand, O. CMOS-based microsensors and packaging. Sens. Actuators A Phys. 2001, 92, 1-9. [CrossRef]

6. Afridi, M.Y.; Suehle, J.S.; Zaghloul, M.E.; Berning, D.W.; Hefner, A.R.; Cavicchi, R.E.; Semancik, S.; Montgomery, C.B.; Taylor, C.J. A monolithic CMOS microhotplate-based gas sensor system. IEEE Sens. J. 2002, 2, 644-655. [CrossRef]

7. Hautefeuille, M.; O’Flynn, B.; Peters, F.H.; O’Mahony, C. Development of a microelectromechanical system (MEMS)-based multisensor platform for environmental monitoring. Micromachines 2011, 2, $410-430$. [CrossRef]

8. Zhang, F.T.; Tang, Z.; Yu, J.; Jin, R.C. A micro-pirani vacuum gauge based on micro-hotplate technology. Sens. Actuators A Phys. 2006, 126, 300-305. [CrossRef]

9. Ivanov, P.; Stankova, M.; Llobet, E.; Vilanova, X.; Brezmes, J.; Gracia, I.; Cane, C.; Calderer, J.; Correig, X. Nanoparticle metal-oxide films for micro-hotplate-based gas sensor systems. IEEE Sens. J. 2005, 5, 798-809. [CrossRef]

10. Semancik, S.; Cavicchi, R.E.; Wheeler, M.C.; Tiffany, J.E.; Poirier, G.E.; Walton, R.M.; Suehle, J.S.; Panchapakesan, B.; DeVoe, D.L. Microhotplate platforms for chemical sensor research. Sens. Actuators B Chem. 2001, 77, 579-591. [CrossRef]

11. Silvestri, S.; Schena, E. Micromachined flow sensors in biomedical applications. Micromachines 2012, 3, 225-243. [CrossRef]

12. Kuo, J.T.W.; Yu, L.; Meng, E. Micromachined thermal flow sensors-a review. Micromachines 2012, 3, 550-573. [CrossRef]

13. Garraud, A.; Combette, P.; Courteaud, J.; Giani, A. Effect of the detector width and gas pressure on the frequency response of a micromachined thermal accelerometer. Micromachines 2011, 2, 167-178. [CrossRef]

14. Chae, J.; Stark, B.H.; Najafi, K. A micromachined pirani gauge with dual heat sinks. IEEE Trans. Adv. Packag. 2005, 28, 619-625.

15. Paul, O.; Baltes, H. Novel fully CMOS-compatible vacuum sensor. Sens. Actuators A Phys. 1995, 46, $143-146$. [CrossRef]

16. Shie, J.S.; Chou, B.C.S.; Chen, Y.M. High-performance pirani vacuum gauge. J. Vac. Sci. Technol. A 1995, 13, 2972-2979. [CrossRef]

17. Ali, S.Z.; Udrea, F.; Milne, W.I.; Gardner, J.W. Tungsten-based SOI microhotplates for smart gas sensors. J. Microelectromech. Syst. 2008, 17, 1408-1417. [CrossRef]

18. Graf, M.; Barrettino, D.; Zimmermann, M.; Hierlemann, A.; Baltes, H.; Hahn, S.; Barsan, N.; Weimar, U. CMOS monolithic metal-oxide sensor system comprising a microhotplate and associated circuitry. IEEE Sens. J. 2004, 4, 9-16. [CrossRef]

19. Yan, G.Z.; Chan, P.C.H.; Hsing, I.M.; Sharma, R.K.; Sin, J.K.O.; Wang, Y.Y. An improved TMAH Si-etching solution without attacking exposed aluminum. Sens. Actuators A Phys. 2001, 89, 135-141. [CrossRef]

20. Tabata, O. PH-controlled TMAH etchants for silicon micromachining. Sens. Actuators A Phys. 1996, 53, 335-339. [CrossRef]

21. Wang, J.Q.; Tang, Z.N.; Li, J.F.; Zhang, F.T. A micropirani pressure sensor based on the tungsten microhotplate in a standard CMOS process. IEEE Trans. Ind. Electron. 2009, 56, 1086-1091. [CrossRef]

22. Wang, J.Q.; Tang, Z.N.; Li, J.F. Tungsten-microhotplate-array-based pirani vacuum sensor system with on-chip digital front-end processor. J. Microelectromech. Syst. 2011, 20, 834-841. [CrossRef]

23. Wang, J.Q.; Tang, Z.A. A CMOS-compatible temperature sensor based on the gaseous thermal conduction dependent on temperature. Sens. Actuators A Phys. 2012, 176, 72-77. [CrossRef] 
24. Sorge, S.; Pechstein, T. Fully integrated thermal conductivity sensor for gas chromatography without dead volume. Sens. Actuators A Phys. 1997, 63, 191-195. [CrossRef]

25. Simon, I.; Arndt, M. Thermal and gas-sensing properties of a micromachined thermal conductivity sensor for the detection of hydrogen in automotive applications. Sens. Actuators A Phys. 2002, 97-98, $104-108$. [CrossRef]

26. Andio, M.A.; Browning, P.N.; Morris, P.A.; Akbar, S.A. Comparison of gas sensor performance of $\mathrm{SnO}_{2}$ nano-structures on microhotplate platforms. Sens. Actuators B Chem. 2012, 165, 13-18. [CrossRef]

(C) 2015 by the authors; licensee MDPI, Basel, Switzerland. This article is an open access article distributed under the terms and conditions of the Creative Commons by Attribution (CC-BY) license (http:/ / creativecommons.org/licenses/by/4.0/). 